\title{
Estomatitis Urémica como Complicación Oral de Insuficiencia Renal Crónica: Reporte de Caso y Revisión de la Literatura
}

\author{
Uremic Stomatitis as an Oral Complication of Chronic Renal Failure: \\ Case Report and Literature Review
}

Pablo Molinare S. ; Kevin Ludwig M. ${ }^{1}$ \& Pablo Quiroz M. ${ }^{1}$

MOLINARE, S. P.; LUDWIG, M. K. \& QUIROZ, M. P. Estomatitis urémica como complicación oral de insuficiencia renal crónica: reporte de caso y revisión de la literatura. Int. J. Odontostomat., 12(3):304-308,2018.

RESUMEN: La Estomatitis Urémica es una lesión oral poco frecuente que se presenta generalmente en pacientes con insuficiencia renal crónica avanzada o no tratada. A continuación, se reporta un caso clínico de un paciente masculino de 22 años de edad que acude a un servicio de urgencia con la presencia de placas blanquecinas indoloras en piso de boca, cara interna de mejilla, y lengua. Las probables causas, presentaciones clínicas, y manejo odontológico son discutidos.

PALABRAS CLAVE: estomatitis urémica, insuficiencia renal crónica, lesiones blanquecinas orales.

\section{INTRODUCCIÓN}

La Insuficiencia Renal Crónica (IRC) corresponde a una condición clínica de daño renal progresivo e irreversible, que provoca una disminución en la tasa de filtración glomerular, y, por consiguiente, un aumento en los niveles plasmáticos de urea (uremia) (Martí Álamo et al., 2011). Si la falla renal no es tratada, puede llegar a estadios más avanzados de la enfermedad, donde se hace necesario emplear técnicas de filtración sanguínea extra renal, principalmente por hemodiálisis (Ross \& Salisbury, 1994). Se presenta generalmente en pacientes mayores de 60 años y posee una predilección por el género femenino (Gavaldá et al., 1999; Szot, 2003; CDC, 2007). Las causas de la IRC más comunes son la diabetes mellitus, hipertensión arterial, glomérulo nefritis, y alteración congénita (poliquistosis renal) (Martí Álamo et al.). El tratamiento médico de la IRC abarca desde cambios en hábitos alimenticios, corrección de complicaciones sistémicas, hasta hemodiálisis y trasplante si la insuficiencia renal es avanzada (Antoniades et al., 2006). Epidemiológicamente, se estima que alrededor del $10 \%$ de la población mundial padece de algunos de los 5 estadios de IRC (Leão et al., 2005). En Chile la prevalencia de IRC tipo III es de un 5,7\%,
IRC tipo IV de un 0,2 \%, y IRC terminal o tipo $\mathrm{V}$ de un 0,1\% (Martí Álamo et al.).

Los signos y síntomas en los pacientes con IRC se conocen como "Síndrome Urémico", ya que la presencia de niveles elevados de urea plasmático afecta a una gran cantidad de tejidos y órganos, pudiendo causar complicaciones neurológicas (neuropatías), cardiovasculares (hipertensión, arritmia), respiratorias, endocrinológicas (hiperparatiroidismo secundario), hematopoyéticas (anemia), gastrointestinal (náuseas y vómitos), urológicas, dermatológicas, craneofaciales y orales (Hovinga et al., 1975; Flores, 2010; Hřibová \& Khazneh, 2014).

La cavidad oral refleja generalmente estados patológicos sistémicos, siendo incluso en algunos casos, donde se suscitan los primeros signos clínicos. El caso de la IRC no es la excepción, el $90 \%$ de los pacientes presenta signos que pueden ser clasificados como objetivos; halitosis urémica, depapilación lingual, petequias, equimosis, ulceraciones, hiperqueratosis, hipocalcificación adamantina. Y subjetivos; xerostomía, disgeusia y boca urente (Hovinga et al.; Jaspers, 1975). 
La Estomatitis Urémica (EU) forma parte del síndrome urémico en pacientes con IRC, sin embargo, es una complicación oral menos frecuente ya que se manifiesta cuando existe una falla renal avanzada o no tratada (Antoniades et al.). Fue mencionada por primera vez en el año 1887 por Etienne Lancereaux y descrita por Barié en 1889 (Zúñiga et al., 2011). Desde sus inicios, se han descrito cuatro presentaciones clínicas en la literatura; ulcerativa, hemorrágica, pseudomembranosa e hiperqueratosica (Barié, 1889; Depner, 2001; Coresh et al., 2005). A continuación, se describe un caso de Estomatitis Urémica en un paciente con IRC tipo IV no tratada. Además, se presenta una breve una revisión de la literatura y se discuten aspectos relativos al rol del odontólogo en el diagnóstico y manejo de esta complicación.

\section{REPORTE DE CASO}

Paciente de sexo masculino, 22 años de edad, acudió a Medicina de la Unidad de Emergencia del Adulto del Hospital G. Fricke, Viña del Mar, por sensación febril, odinofagia y disfagia de dos semanas de evolución. Como antecedente médico, presentó una poliquistosis renal bilateral diagnosticada a los 4 años, la que fue controlada por nefrólogo sólo hasta los 18 años. Se realizó un examen físico, y examen hematológico por sospecha de una alteración sistémica del paciente, donde se encontró un valor de creatinina de 10,4 $\mathrm{mg} / \mathrm{dl}$, urea de $379 \mathrm{mg} / \mathrm{dl}$, y eritrocitos de 2,69 millones $/ \mathrm{mm}^{3}$ (Tabla I). Se derivó a especialista maxilofacial por la presencia de placas blanquecinas en la mucosa oral.

En la anamnesis odontológica, el paciente relata que además presenta disgeusia, sensación de ardor, mal aliento y manchas blancas en la boca hace 8 meses atrás.

Al examen intraoral se encontraron placas blanquecinas indoloras, no desprendibles al raspado en toda la superficie de piso de la cavidad oral, vientre lingual, márgenes laterales, dorso lingual, y cara interna de mejilla, esta última recubierta parcialmente por una "corteza amarillenta" (Figs. 1, 2, y 3).

Fue derivado a medicina interna en pos de estudiar y estabilizar el cuadro sistémico. Se diagnosticó finalmente con un cuadro insuficiencia renal tipo IV por lo que se decidió hospitalizar al paciente para comenzar tratamiento de hemodiálisis. Junto al diagnóstico médico del paciente, exámenes hematológicos y

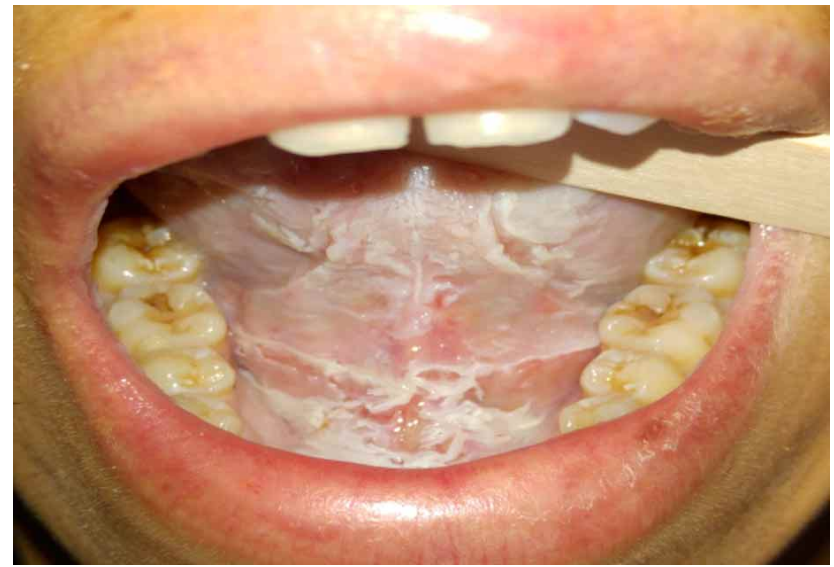

Fig. 1. Apariencia clínica de lesiones blanquecinas en toda la superficie del piso de la cavidad oral y vientre lingual.

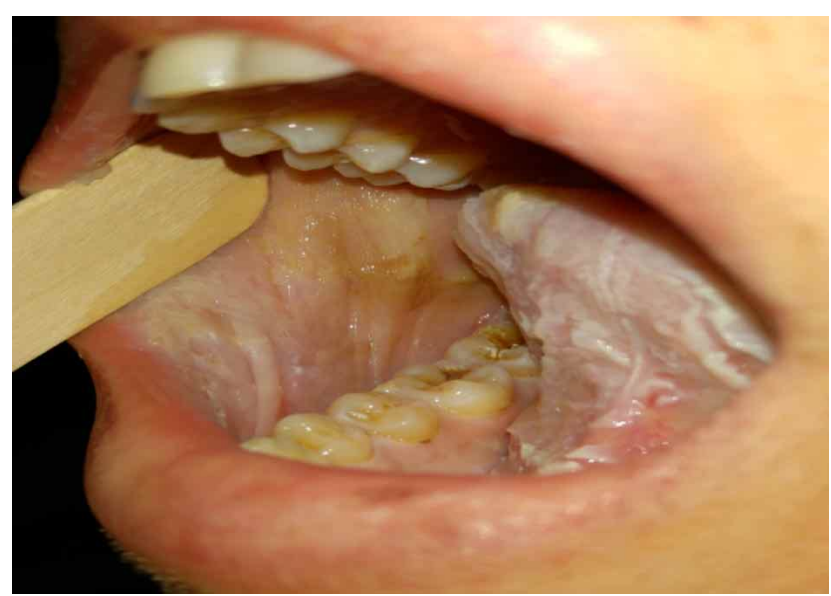

Fig. 2: Cara interna de mejilla antes del tratamiento médico.

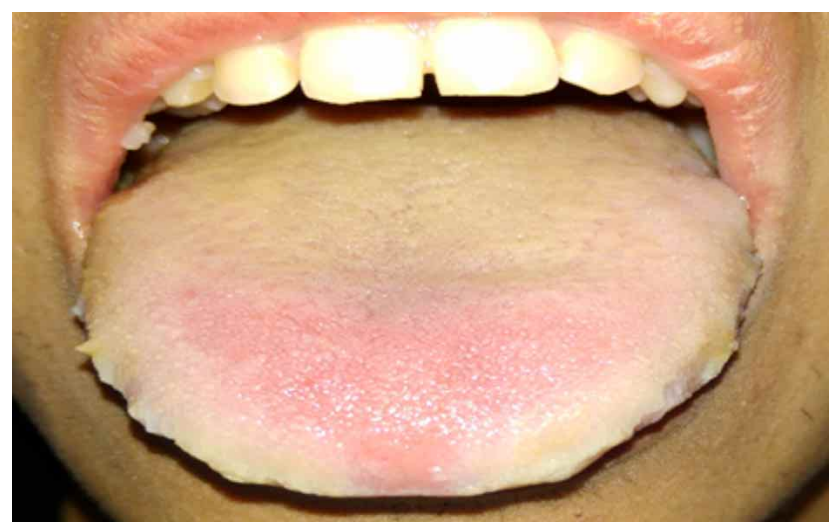

Fig. 3. Dorso y márgenes laterales de lengua antes del tratamiento médico.

examen clínico intraoral, se realizó un diagnóstico odontológico presuntivo de estomatitis urémica.

Se comenzó el tratamiento médico con amoxicilina $500 \mathrm{mg}$ cada $8 \mathrm{~h}$, metronidazol $500 \mathrm{mg}$ cada 8 h, suero fisiológico de 1000 cc diarios, ácido 
Tabla I. Exámenes hematológicos y perfil bioquímico del paciente antes y después del tratamiento médico.

\begin{tabular}{llc}
\hline Parámetro & Antes del tratamiento médico & Después del tratamiento médico (7 días) \\
\hline GR & $2,99$ (normal $=4,3-5,7)$ & 2,94 \\
GB & 5000 & 3200 \\
NEU & 70 & 74,7 \\
LIN & 20 & 16,6 \\
MONO & 6,6 & 7,3 \\
HGB & 8,9 & 8,7 \\
HTC & 26,6 & 26 \\
PQT & 137 (normal=150-400) & 97 \\
UREA & $379($ normal=19-43 mg/dl) & 186 \\
CREAT & $10,4($ normal $=0,66-1,25 \mathrm{mg} / \mathrm{dl})$ & 8,8 \\
FOSF. ALC & $196($ normal $=38-126 \mathrm{mg} / \mathrm{dl})$ & 173 \\
K & 4,3 (normal=3,5-5,1) & 3,4 \\
Na & $136($ normal $=136-146)$ & 139 \\
PT & $71,5($ normal $=70-130)$ & 71 \\
TTPA & 26,5 (normal=20-30) & 26,5 \\
\hline
\end{tabular}

GR: glóbulos rojos, GB: glóbulos blancos, NEU: neutrófilos, LIN: linfocitos, MONO: monocitos, HGB: hemoglobina, HTC hematocrito, PQT: plaquetas, UREA: urea, CREAT: creatinina, FOSF.ALC: fosfatasa alcalina, K: potasio, Na: sodio, PT: protrombina, TTPA: tiempo de tromboplastina parcial.

fólico $5 \mathrm{mg}$ diarios, carbonato de calcio $500 \mathrm{mg}$ diarios vía oral, gluconato de calcio $10 \mathrm{~mL}$ c/8 h y dieta

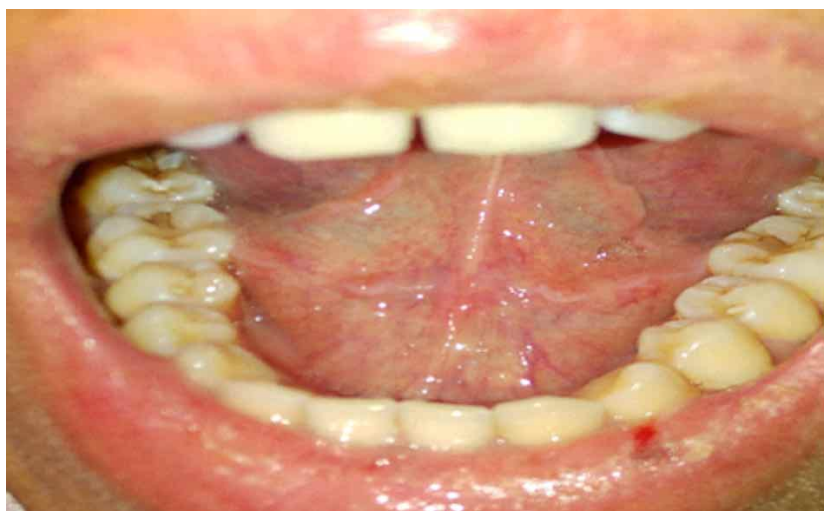

Fig. 4. Piso de la cavidad oral a los 7 días de tratamiento médico.

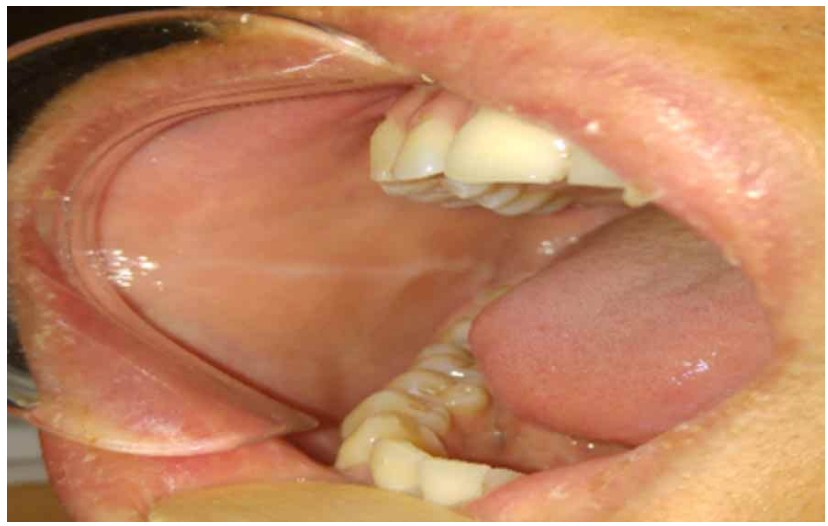

Fig. 5. Cara interna de mejilla a los 7 días de tratamiento médico. hipoproteica. El control odontológico se realizó a los 7 días y el paciente ya se había realizado 2 hemodiálisis. Al examen clínico, las placas blanquecinas habían remitido en su totalidad y el paciente ya no presentaba odinofagia, disgeusia, ni sensación de ardor (Figs. 4, 5 y 6). Al examen hematológico presentó una creatinina de $8,8 \mathrm{mg} / \mathrm{dl}$, y una urea de $186 \mathrm{mg} / \mathrm{dl}$.

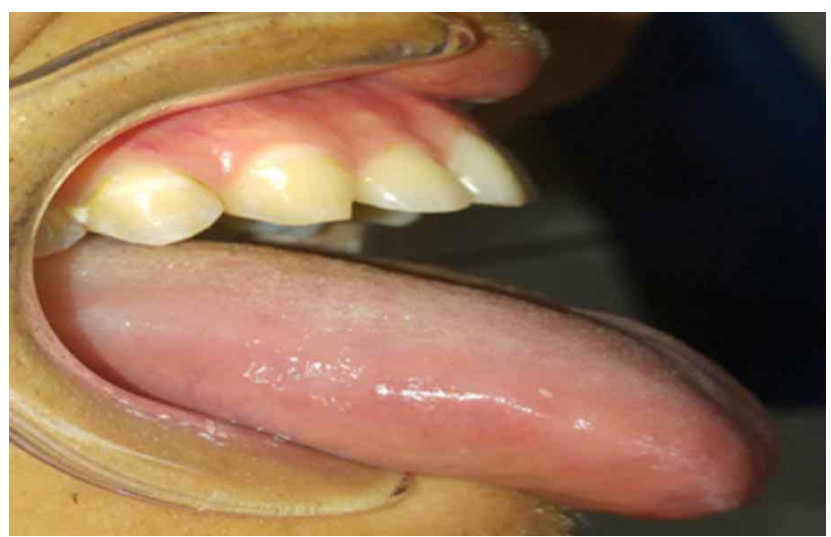

Fig. 6. Borde lateral de lengua a los 7 días de tratamiento médico.

\section{DISCUSIÓN}

La EU es una manifestación oral poco frecuente que aparece en pacientes con insuficiencia renal crónica avanzada o en falla renal sin diagnóstico ni tratamiento, donde escasos casos han sido reportados en la literatura (Tabla II) (Barié). 
Tabla II. Casos de estomatitis urémica reportados en la literatura.

\begin{tabular}{lll}
\hline Estudios & $N^{\circ}$ de casos & Tipo \\
\hline Hirtz, 1902 (Zúñiga et al., 2011) & 2 & 1 Ulcerativo; 1 Pseudomembranoso \\
Claude, 1903 (Zúñiga et al., 2011) & 1 & Ulcerativo \\
Meyerson, 1927 (Zúñiga et al., 2011) & 5 & 4 Pseudomembranoso, 1 Ulcerativo \\
Hempstead \& Hench, 1930 (Zúñiga et al., 2011) & 4 & 3 ulcerativo;1 Pseudomembranoso \\
Bereston \& Keil, 1941 (Zúñiga et al., 2011) & 1 & Ulcerativo \\
Black, 1942 (Zúñiga et al., 2011) & 1 & Ulcerativo \\
Hovinga et al., (1975) & 13 & Ulcerativo \\
Jaspers, (1975) & 1 & Pseudomembranoso \\
McCreary et al., (1997) & 1 & Ulcerativo-Hiperqueratódsico \\
Leão et al., (2005) & & Hiperqueratosica \\
Mini et al. (2015) & 1 & 1 Hiperqueratosica 1 Pseudomembrano \\
Presente caso (2016) & 2 & Hiperqueratosica \\
\hline
\end{tabular}

La etiología de la Estomatitis Urémica todavía se desconoce, sin embargo, se sugiere que puede ser consecuencia de elevados niveles de amonio en la saliva. (Depner; Coresh et al.) La ureasa provoca la hidrólisis de la urea para formar amonio y carbamato (Barié; Coresh et al.). Esto ocurre cuando la concentración de urea intraoral excede los $30 \mathrm{mmol} / \mathrm{L}$ (en paciente sano la saliva estimulada contiene de 2 a 6 $\mathrm{mmol} / \mathrm{L}$ de urea) (Barié). Según una revisión realizada por Hribová \& Khazneh, las bacterias más relevantes productoras de ureasa son Estreptococos, Helicobacter pylori, Proteus, Klebsiella, Pseudomonas y Estafilococos. Siendo característico para la cavidad bucal el caso de la Estreptococos Salivarus, bacteria colonizadora de estructuras orales que utiliza el nitrógeno proveniente de la urea como recurso primario para su proliferación (De Rossi \& Glick, 1996). La liberación de amonio parece tener el mayor impacto en termino de patogénesis, al ser toxico para las células, es por esto que las lesiones podrían considerarse una quemadura química. (Depner; Coresh et al.).

Otra posible causa de cambios en la mucosa asociado a la uremia, es la susceptibilidad a la hemorragia, lo cual es común en pacientes con uremia, causando un descenso en la vitalidad de los tejidos afectados y predisposición a infecciones bacterianas, que pueden resultar en ulceraciones y formación de pseudomembranas (Depner; Coresh et al.).

En relación a las presentaciones clínicas de la EU, se describen 4 tipos: a) Ulcerativa, b) Hemorrágica, c) Pseudomembranosa y d) Hiperqueratosica. El tipo ulcerativo es el más común, se caracteriza por forma- ción de ulceras dolorosas y zonas eritematosas en la mucosa (Barié). En diversos estudios histopatológicos del tipo ulceroso, se describieron extensas áreas de necrosis epitelial y la presencia de una infiltración inflamatoria densa, no específica en el tejido conectivo (PMNs, y linfocitos principalmente), a veces extendidos en el epitelio. Por lo general, las levaduras o pseudomycellium no son encontradas (Szot). La EU hemorrágica se caracteriza por sangrado especialmente de las encías. Esta tendencia al sangrado se debe a la inhibición de la función plaquetaria (Barié; Coresh et al.). La forma pseudomembranosa se presenta con formaciones de membranas sobre un fondo de mucosa eritematosa, suelen ser bastante dolorosas y generalmente se presentan en altos niveles de Urea en un corto tiempo (Barié). La forma hiperqueratósica presenta menos casos reportado, ya que se asocia a un largo periodo de falla renal, generalmente demuestra a la observación histopatológica una acantosis con una zona paraqueratósica gruesa en la superficie y una capa de queratinocitos ovalados por debajo (Svirsky et al., 1998; Depner). El presente corresponde a un caso de EU hiperqueratósica dada su apariencia clínica y su persistencia a pesar de la ejecución de pruebas de raspado.

La confirmación diagnóstica de la EU se basa en los signos y síntomas clínicos, historia médica, análisis de sangre (niveles de creatinina y urea), y manchas microbianas especiales de frotis de tejidos. Los hallazgos microscópicos de la EU son inespecíficos, por lo que el estudio histopatológico sólo estaría indicado cuando la historia médica del paciente no contribuye a su diagnóstico o bien, cuando las lesiones 
orales simulan a otras lesiones blancas conocidas caracterizadas por placas blancas asociadas a formación de queratina, no desprendibles al raspado indicadas en la Tabla III. El diagnóstico diferencial dependerá de su presentación clínica e incluye enfermedades del tejido conectivo y vesiculoampollosas, infecciones microbianas/ micóticas, y deficiencias de vitaminas (Barié).

El tratamiento de la EU es principalmente médico enfocado en el mejoramiento de la insuficiencia renal, ya que numerosos estudios han demostrado que las lesiones intraorales son resistentes al tratamiento local siempre y cuando la concentración de urea en la sangre permanezca alta (Barié). El tratamiento odontológico tiene como objetivo ser un apoyo al tratamiento médico, enfocándose en la mejoría de la higiene bucal (eliminación del cálculo dental que puede contener enzimas ureasas), colutorios antisépticos, y la utilización de geles anti fúngicos/antimicrobianos si es necesario (Barié; Depner; Coresh et al.). Algunos autores hasta recomiendan el uso de enjuagues con peróxido de hidrogeno ya que contribuye a la eliminación de las bacterias anaerobias productoras de amonio. Las lesiones orales no debiesen durar más allá de 2 a 3 semanas luego de la disminución de la uremia.

MOLINARE, S. P.; LUDWIG, M. K. \& QUIROZ, M. P. Uremic stomatitis as an oral complication of chronic renal failure: Case report and literature review. Int. J. Odontostomat., 12(3):304-308, 2018.

ABSTRACT: Uremic stomatitis is a rare oral lesion that usually occurs in patients with advanced or untreated chronic renal failure. Here we report a case of a 22-year-old male patient who comes to an emergency department with the presence of painless whitish plaques on the floor of the mouth, internal cheek face, and tongue. Probable causes, clinical presentations, and dental management are discussed.

KEY WORDS: uremic stomatitis, chronic renal failure, whitish oral plaques.

\section{REFERENCIAS BIBLIOGRÁFICAS}

Antoniades, D. Z.; Markopoulos, A. K.; Andreadis, D.; Balaskas, I.; Patrikalou, E. \& Grekas, D. Ulcerative uremic stomatitis associated with untreated chronic renal failure: report of a case and review of the literature. Oral Surg. Oral Med. Oral Pathol. Oral Radiol. Endod., 101(5):608-13, 2006.

Barié, E. De la stomatite uremique. Arch. Gen. Med., 2:415-32, 1889.

Centers for Disease Control and Prevention (CDC). Prevalence of chronic kidney disease and associated risk factors--United States, 1999-2004. MMWR Morb. Mortal Wkly. Rep., 56(8):161-5, 2007.

Coresh, J.; Byrd-Holt, D.; Astor, B. C.; Briggs, J. P.; Eggers, P. W.; Lacher, D. A. \& Hostetter, T. H. Chronic kidney disease awareness, prevalence, and trends among U.S. adults, 1999 to 2000. J. Am. Soc. Nephrol., 16(1):180-8, 2005.

De Rossi, S. S. \& Glick, M. Dental considerations for the patient with renal disease receiving hemodialysis. J. Am. Dent. Assoc., 127(2):211-9, 1996.

Depner, T. A. Uremic toxicity: urea and beyond. Semin. Dial., 14(4):246-51, 2001.

Flores, H. J. C. Enfermedad renal crónica: epidemiología y factores de riesgo. Rev.
Tabla III Placas blancas no desprendibles al raspado. DD con EU.

\begin{tabular}{|c|c|}
\hline \multicolumn{2}{|l|}{ Causas locales } \\
\hline & Quemaduras. \\
\hline & Queratosis (Friccional del fumador) \\
\hline & Injertos \\
\hline & Cicatrices \\
\hline \multirow[t]{3}{*}{ Causas congénitas } & Gránulos de Fordyce \\
\hline & Leucoedema \\
\hline & Disqueratosis Hereditaria \\
\hline \multirow{5}{*}{$\begin{array}{l}\text { Causas inflamatorias; } \\
\text { infecciosa }\end{array}$} & \\
\hline & Micosis (Ejemplo: Candidiasis) \\
\hline & Viral; Hiperplasia pilosa \\
\hline & Papiloma virus Humano \\
\hline & Bacteriano (Ejemplo placa sifilítica) \\
\hline \multirow[t]{2}{*}{ No infecciosa } & Liquen plano \\
\hline & Lupus eritematoso \\
\hline
\end{tabular}

Med. Clin. Condes, 21(4):502-7, 2010.

Gavaldá, C.; Bagán, J.; Scully, C.; Silvestre, F.; Milián, M. \& Jiménez, Y. Renal hemodialysis patients: oral, salivary, dental and periodontal findings in 105 adult cases. Oral Dis., 5(4):299-302, 1999.

Hovinga, J.; Roodvoets, A. P. \& Gaillard, J. Some findings in patients with uraemic stomatitis. J. Maxillofac. Surg., 3(2):125-7, 1975.

Hrbová, P. \& Khazneh, E. An overview of urease and its relation to the ureolytic bacteria and the search for new urease inhibitors. Ceska Slov Farm., 63(6):248-52, 2014.

Jaspers, M. T. Unusual oral lesions in a uremic patient. Review of the literature and report of a case. Oral Surg. Oral Med. Oral Pathol., 39(6):934-44, 1975.

Leão, J. C.; Gueiros, L. A. M.; Segundo, A. V. L.; Carvalho, A. A. T.; Barrett, W. \& Porter, S. R. Uremic stomatitis in chronic renal failure. Clinics (Sao Paulo), 60(3):259-62, 2005

Martí Álamo, S.; Gavaldá Esteve, C. \& Sarrión Pérez, M. G. Dental considerations for the patient with renal disease. J. Clin. Exp. Dent., 3(2):e112-9, 2011.

McCreary, C. E.; Flint, S. R.; McCartan, B. E.; Shields, J. A.; Mabruk, M. \& Toner, M. E. Uremic stomatitis mimicking oral hairy leukoplakia: report of a case. Oral Surg. Oral Med. Oral Pathol. Oral Radiol. Endod., 83(3):350-3, 1997

Mini, M. M.; Prasad, T. S. \& Thomas, V. Uremic Stomatitis: Report of Two Cases. Oral Maxillofac. Pathol. J., 6(2):636-8, 2015.

Ross, W. F. 3rd. \& Salisbury, P. L. $3^{\text {rd }}$. Uremic stomatitis associated with undiagnosed renal failure. Gen. Dent., 42(5):410-2, 1994.

Svirsky, J. A.; Nunley, J.; Dent, D. \& Yeatts, D. Renal disease. Dental and medical considerations of patients with renal disease. J. Calif. Dent. Assoc., 26(10):763-70, 1998.

Szot, M. J. La transición demográfico-epidemiológica en Chile, 1960-2001. Rev. Esp. Salud Pública, 77(5):605-13, 2003.

Zúñiga, S. M. C.; Müller, O. H. \& Flores, O. M. Prevalencia de enfermedad renal crónica en centros urbanos de atención primaria. Rev. Med. Chile, 139(9):1176-84, 2011.

Dirección para correspondecia:

Dr. Pablo Molinare Severino

Cirugia Maxilofacial de la Unidad de Emergencia del

Adulto

Hospital G. Fricke

Santiago - CHILE

Recibido : 19-04-2017

Aceptado: 01-06-2018

Email: pablo.molinare@gmail.com 